\title{
DEVELOPMENT OF A SMART WORKSTATION BY USING AR TECHNOLOGY
}

\author{
Kätlin Värno, Kashif Mahmood, Tauno Otto \& Vladimir Kuts
}
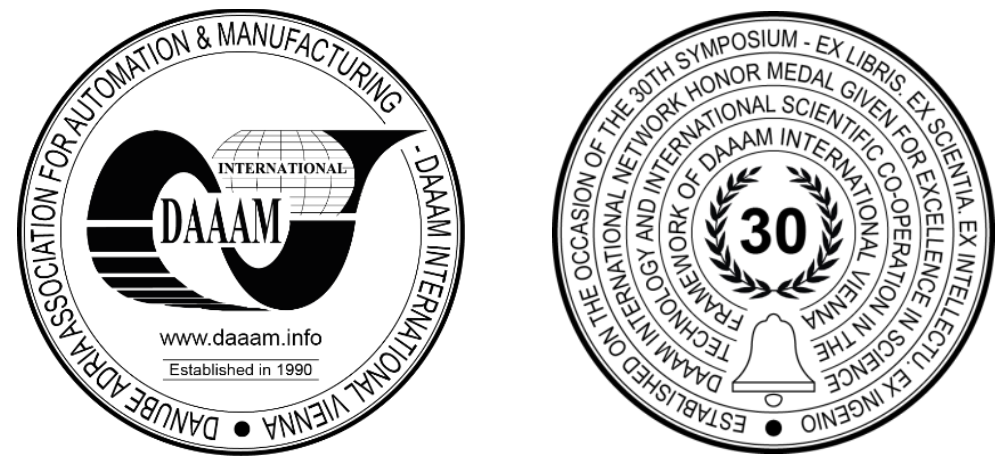

This Publication has to be referred as: Varno, K[atlin]; Mahmood, K[ashif]; Otto, T[auno] \& Kuts, V[ladimir] (2019). Development of a Smart Workstation by Using AR Technology, Proceedings of the 30th DAAAM International Symposium, pp.1061-1067, B. Katalinic (Ed.), Published by DAAAM International, ISBN 978-3-902734-22-8, ISSN 1726-9679, Vienna, Austria

DOI: $10.2507 / 30$ th.daaam.proceedings.148

\begin{abstract}
A recent development in the area of digital manufacturing demands the manufacturing industry to adopt industry 4.0 technologies to be competitive in the global market. Product development processes are becoming complex as products are getting more versatile and product variations increase the trend of mass customization. Therefore, manufacturing processes need to be more systematic and well-organized to be economically competent and efficient at the same time. The application of Virtual Reality (VR) and Augmented Reality (AR) technologies may provide effective and innovative solutions in the manufacturing work environment. Especially, AR can help to speed up the assembly process by facilitating the assembly workers to assemble a product without involving into the detailed manual instructions. It may also use as a training aid for new workers to get familiar with the assembly process by visualization and provide easiness in the work. This paper aims to analyse the rationality of digitalization in the manufacturing by using AR technology for a pilot assembling workstation of a cooling unit. An approach to creating digital instructions application by AR was described and the relevance of the approach has shown through a case study.
\end{abstract}

Keywords: Digital technology; VR and AR; smart workstation; monitoring and application design.

\section{Introduction}

Nowadays it is necessary to keep pace with fast technology developments in various industries like in the fields of energy, wood, construction and other important areas of society, where the aim is to be more cost-effective and innovative. Digitalization is now one of the largest investment categories in the world which helps to test and develop the best possible way to save resources while ensuring safety in the work environment.

Industrial sectors that are integrated with virtual and augmented reality have been an important topic for many years. The Gartner Hype Cycle chart [1] can be seen in the Fig.1 which gives an overview of the stage and evolution of different technology solutions over time. The trend line shows that, within 2 to 5 years, companies should embrace virtual assistants, smart working environment become commonplace in the 5 to 10 -year perspective. The chart contains 35 technologies divided into five categories. In the future, potential technological developments and applicability are reviewed for the next 10 years. It is monitored by business and technology managers who observed rapidly evolving 
innovation which has an impact on the workforce, cooperation between partners, product manufacturing and customer service [1].

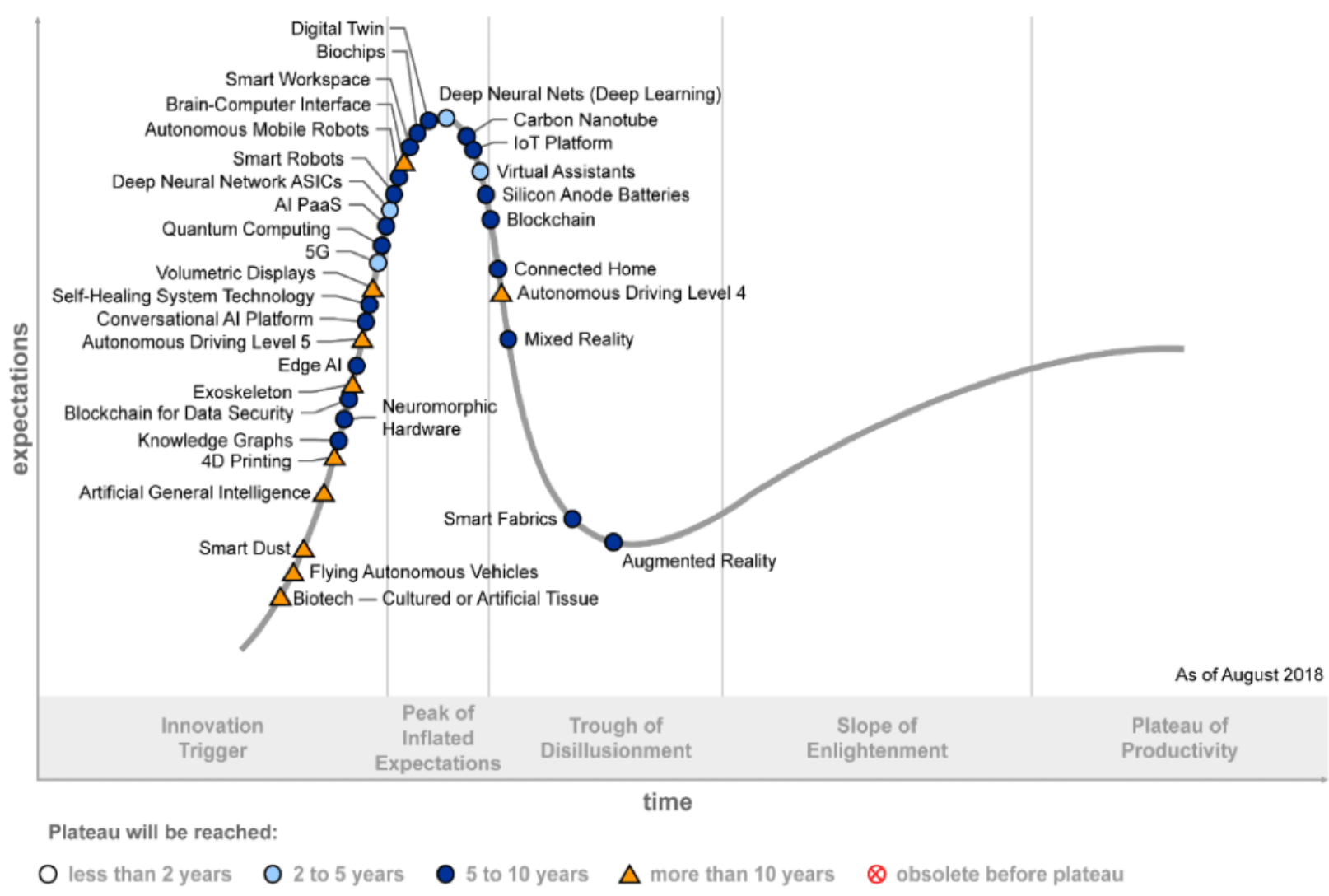

Fig. 1. Emerging technologies portrayed by Gartner as Hype Cycle [1]

Grand View Research shows a virtual technology upward trend of the global market for virtual prototyping and indicating an increasing need for virtual tools. At the moment, bigger ICT companies have invested the most in AR and VR sensor devices where they own several virtual patents. Virtual prototyping is a software-based process that helps to build simulations and test the original version of the products in a virtual reality environment. Businesses and industrial manufacturers are preparing for a transition through a variety of virtual tests to save time and costs (as opposed to physical testing). Also, virtual prototype solutions improve product testing accuracy and increase ergonomics and usability. In particular, it is necessary to satisfy the general demand of end-users, e.g. electronic devices. Satisfying demand creates new opportunities to be innovative and provide even more efficient solutions that can compete with other market participants while increasing profits. However, the above factors call for the expansion of the global market in the forecast period [2], [3].

The purpose of this paper is to analyze the feasibility of emerging technologies such as Augmented Reality technology in the manufacturing industry for an assembly process. An overview about the suitability of digital technologies in the industrial sector was described, the study focused on the creation of digital work instructions through the AR application to support an assembly process and to develop a smart workstation. Moreover, a case study performed at TalTech IVAR laboratory for testing purpose of the developed AR application and to demonstrate the results of the smart workstation.

\section{Literature Review}

Industry 4.0 leads revolutionary industrial development, bringing together several areas: the cyber-physical system (CPS), the Internet of Things (IoT) and cloud data processing. IoT uses communication and data exchange between objects and systems [4]. CPS has more reliance on software-based information sharing than the IoT mechanism. Cloud data processing has an even greater spectrum that combines the entire product life cycle, sharing information with other parties such as customers [5].

The greatest need is for communication between devices and systems that collect information and which in turn $\underline{\underline{\text { generates and improves knowledge. Through the information exchange process, the smart workplace has finally been }}}$ 
reached, helping to be economical and smart enough to make the work more efficient. To design a smart workplace, it is necessary to find the right tools to make the work environment more flexible [6] and VR/AR technologies have the potential to offer flexibility and capability.

The biggest difference between virtual and augmented reality is the surrounding environment. VR technology allows to go into the virtual world, but with augmented reality, information displaying to the physical world. AR has also been developed for use in industry area, which can simplify workflows. In mixed reality, physical and virtual are visually blended by subjecting reality to computer-generated content [5], [7].

\subsection{Augmented Reality as an Emerging Technology}

Augmented Reality is rapidly becoming a new user interface for factories, service technicians and engineers. The realtime information can be display to the real world, complemented by pre-processed information on the computer. The device shows a composite view, where the real image is viewable by the user, and the virtual image is displayed by a device, such as a computer, which connects to it. The application visualization appears on the screen, which can be monitored through smart glasses, tablet or other visualization electronic devices [8], [9].

The entire industry is forced to make innovations soon with the help of an expanded reality that would save money and increase efficiency. In particular, the expanded reality is seen as a simulation technology that does not aim at automation, but rather a human-machine relationship development technology that helps to improve the quality and efficiency of work [8]. It is possible to check the correctness and positioning of the components that are applied in advance for each new step. This reduces the risk of doing something wrong, either by choosing the right component or tools. When visualizing information and objects in the real world, 3D models give a better idea of the assembly module and help to perceive the three-dimensional final result [8], [10].

The use of AR creates opportunities to reduce the time spent on work. It is possible to follow the instructions faster. Then there is no need to look a guidebook. Usually, the guides are long and monotonous. Using conventional solutions, it is difficult for the compiler to read and monitor the assembly processes in text format when performing new tasks. AR gives the ability to easily update guides. The created application encompasses all the 3D models of the module by selecting exactly the right material to be displayed on the screen with 3D images, quantities and instructions. Augmented Reality is a good tool for those who need help in coaching. Using digital guidance, the use of the AR application influences the price formation of the entire product, because assembly work is one of the most expensive of the entire work process [9], [10].

For example, there are good study cases which went very well. First of all, there is a warehouse AR application integration. Warehouse process digitalizing helps to see the input and output of products. There is much better inventory management which enables constant awareness of the available stock. Users can navigate and act more efficiently whenever a request is sent to the warehouse [11].

The second case study from Volvo shows that engine quality control and assurance in manufacturing plants are subject to rigorous quality checks which need high experienced technicians. Training new technicians means five-week work, who should do complex inspection procedures, which adds to the overall cost of quality. There is also a need to establish and maintain consistent data flow and systems connections to create operational efficiency across the value chain. Using a Vuforia augmented reality experience, which is used also in this heatsink assembly study case, operators can quickly recall the most up-to-date configurations in 3D to ease the burden of sorting through stacks of paper, creating gains in productivity, quality control, and overall process efficiency. At the same time removing the cost and risk of the paperbased approach, the solution also enables to avoid specific defects through the using AR experience [12]. Future industry assembly work expects these key factors: higher quality and maintenance, faster and an efficient work, less rework, money and energy waste [13].

\subsection{Role of Machine Vision}

Machine vision technology is a combination of hardware and software that helps devices deliver operational instructions based on image processing. The main components of the machine vision system are camera, lighting, lenses, image sensor, image processing software and user interface. The lighting illuminates the objects to be seen so that they can be distinguished through the camera image [13]. Object visualization is based on image processing and computational intelligence based on neural networks, thereby improving AR and VR applications that define and design 3D virtual models. When using augmented reality, machine vision is mainly related to video tracking. During the tracking phase, important markers, optical image communities, or points of interest are identified with the camera. Machine tracking uses a feature or edge detection, which is called function and model-based tracking, to define objects. Function-based model tracking includes a 2D image function and their 3D environment framework coordinate, while model-based tracking uses object models based on the detection of CAD drawings or 2D models. Once the object is detected, the data is sent to the reconstructed real-world coordinate system. The application of such system are reached to industries and Industrial Augmented Reality (IAR) solutions are build and tested for different industrial case studies [14], [15]. 


\subsection{Supportive Simulation Technologies for AR}

The logical architecture and user-friendly interface for mobile devices, such as a tablet, are important in developing the entire simulation to support AR solution. The interface displays information about the process being supervised, malfunctions, and other necessary information about assembly work. Creating an augmented reality application for subassembly workplace requires the following simulation technologies: computer, input devices, camera, screen (visualization), application, computer software.

Input simulation application devices are divided into categories: attach to the head, handheld devices, or screens displayed in the environment. These may include specially designed glasses, headsets, or tablets. Tablets are preferred for smartphones to ensure broader visualization capability or greater image creation and processing of information power. The problem is space usage, which is important for every job because larger screens require space. Handheld devices limit operator efficiency while the first tablet PCs are more widely used compared to AR tablet PCs and future users are more familiar with the use of technology than AR glasses, which are currently accompanied by a tight battery charge and overheating. The impact of eyeglasses on human health plays a major role. At the moment, these devices are still heavy, especially the HoloLens spectacles, but the AR and VR HoloLens 2 series have been designed to overcome the difficulty distribution on the forehead, allowing them to work longer and avoid fatigue. In addition, eye irises and hand movements are defined. The field of view has been magnified, where the first version had solved the field of view with only small window boxes [16], [17].

Health risks include anxiety or stress caused by the nature of extended reality after using technology. Headaches, dry eyes, and tiredness may occur. User-friendliness is an important aspect, especially when using a touch screen device. Another way to provide feedback on application communication is to integrate sound and vibration. When the module is assembled and an error occurs, the application can know which output is a special sound or tablet vibration signal that is generated at the time of holding the hand, ensuring that the user of the application responds faster and the work process is, therefore, more efficient. When considering variants at the factory, vibration generation is more relevant because noise can make the sound unnoticed. This allows the compiler to notice the exact location of the error on the screen faster with visual marking [18].

\section{Approach to Develop an Application}

Case study approach as a research method was used to demonstrate the findings of AR application. Likewise, based on the literature review a CESAR approach was established as shown in Fig. 3, which helps to develop an AR application for the selected use-case. To design a smart work-place, it is necessary to find the right tools to make the work environment more flexible, and VR/AR technologies have the potential to offer flexibility and capability. First of all, for planning the digital assembly workstation, there is a need to define manufacturing needs and opportunities. The evaluation helped to choose tools and techniques, which enabled to create Augmented Reality solution for the defined needs.

-Define the needs of particular case study to implement AR technology

Evaluation Unity and Vuforia platforms were used - Selection of tools and techniques for development of AR application

Hardware \& Software: Tablet and glasses, 3ds Max, SolidWorks, Visual
Studio, etc.
Assemblying times were compered

- Feasibility of developed AR application through KPIs such as: cost and time efficiency

Results

- Outcomes and Future actions

Fig. 2. CESAR approach for AR application development 
The AR application consists of Unity (game engine) [19], [20] and Vuforia [21], [22], which provides to display the augmented reality picture. Unity is designed to create interactive content. The development environment is suitable for both operating systems (Windows and Mac OS X), which creates an Android platform application. The gaming engine allows using 2D and 3D models, which applies physical laws, sound, scripting, animations, artificial intelligence, scene graphics, and more. Scripting is a different form of conventional programming, and Visual Studio helped to manage it. Using the scripts allows to define GameObject behaving and also additional components that need to communicate with each other. On the other hand, Vuforia can activate the AR solution. Vuforia Engine 8.1 gives the possibility to develop the application with AR tools, where the most important is to the AR camera option, which visualizes the necessary information based on the augmented reality. It supports the choice of devices for smartphones, tablets, head-to-head devices to provide the most dynamic detection of objects, perception of images and 3D models.

An Android tablet is being used to digitalize the workstation, which is a necessary device to use the application. Parameters such as screen resolution, screen size, battery life, operation system, and internal memory capacity were considered to select the device. As Unity was used to create user interface, therefore, 3D models and markings were needed that generates functional scripts. First of all 2D drawings of product or parts were obtained which are supposed to be assembled and modeled in a 3D software. After transferring the models into the Unity program, a 3D computer graphics program was used that supports Unity and suitable for creating animations, games, and visuals. It was done to reduce the polygons and to optimize the viewing angle of objects.

The steps of operating the AR application are illustrated in Fig. 4. At start, a list of parts' material, an image of the material to be installed as animation and a text box were appeared. When choosing the material, animation showing correct position of material. Moreover, a list provides an overview of which part of the material is being installed at a certain point in time. The image of the material to be installed and displayed in the camera view helps to clarify the properties of the material to be selected and the installation method. The text box contains additional information about the material code and the installation of observations. By opening the application, a QR code identifier shows specific product, by selecting "go to next" gives the script an ability to select the material from the list. Then, the primary guide for the user in the text box was appeared, that guides how to place the specific product and a sub-assembly material component is animated in the camera view. At the end of the installation, press Confirm Part Placed, which signals to the script that the action has been completed and automatically redirecting the next material to the selection and instruction.

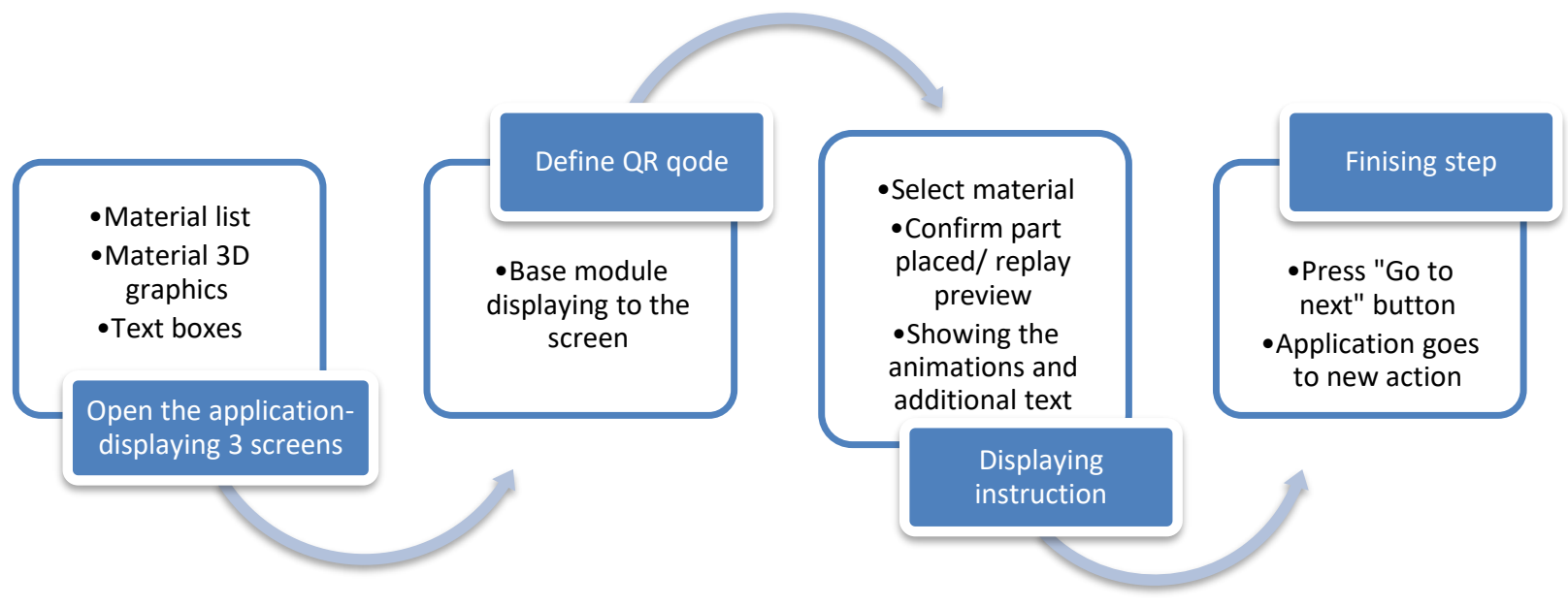

Fig. 3. Working steps of AR application

\section{Verification and Testing of AR Application}

To test the prototype solution, there is a need to set up a tablet and download the application. The test performed in TalTech IVAR laboratory by a student who has not previously been involved in the assembly work of the selected product work and have not seen the guide before. The testing of the AR application can be depicted in Fig. 5. The time factor is measured and observed over the experiment. The assembly of the module (selected product) does not require the use of power tools or other accessories (e.g., cleaning cloth, thermal paste). The components were spread out on the desktop in small cardboard boxes which were marked with the respective component codes. Following the paper instruction manual, the assembly time was 28 minutes, and the digital working (use of AR app) time was 27 minutes. The errors in the paper instruction for example were misunderstanding the image because it was not understood the need to turn around subassembly components. Besides, there was confusion about the colour code, which resulted in a change in an assembly 
line. There are no errors in assembly work with the AR application. Moreover, paper instruction tracking was more difficult. The order of the colour codes had to be followed very precisely. Conversely, tablet component movements were shown with animations that made tracking materials easier. As a result of the test placing, traceability and user-friendliness has improved, which helps to navigate better in the order of assembly.

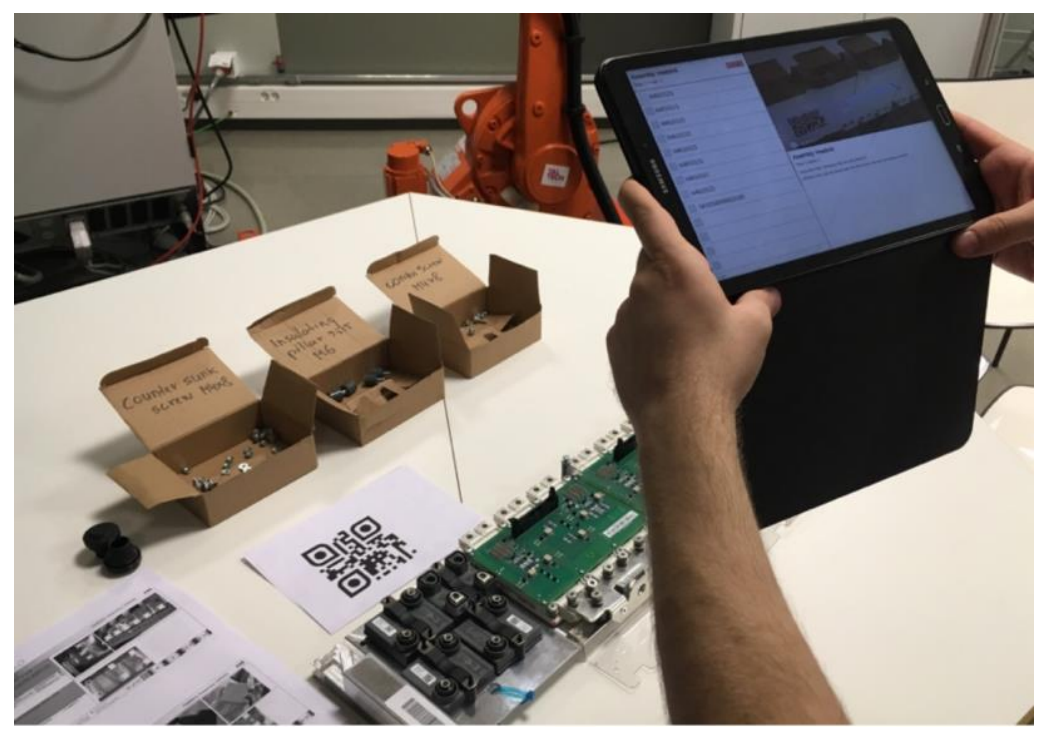

Fig. 4. Testing of AR application

\subsection{Implications of the AR Application}

The goal is not only automation and digitalization of a workplace (workstation), but also to contribute in human-machine relationship development technology that helps to improve the quality and efficiency of work.

The impact of eyewear on human health plays a major role. The devices used for AR application are still heavy, but for example the HoloLens 2 series has been developed to solve the weight distribution on the forehead, allowing them to work longer and avoid fatigue. Also, eye movements are defined as hand movements. The field of view has been magnified, where the first version had a resolved field of view with only small windows.

The other health risks can also include anxiety or stress which caused by using augmented reality. Headaches, dry eyes and tiredness may occur. User-friendliness is an important aspect, especially when using a touch screen display.

Another way to provide application interaction feedback is to integrate tablet vibration. When an assembly error occurs, the application can let know with special tablet vibration signal as the user hold tablet with hands, ensuring the application's user is more responsive and therefore more efficient. Also, the machine vision can quickly detect error location by the visual marking on the screen. The result is easier traceability of the workflow and a faster module assembly process, increasing the quality of assembly work, reducing at the same time higher financial and time costs. Factors such as user-friendliness, quality and system logic, rapid learning and safety were considered.

Further enhancements could be adding to the application more specific components of properties. Adding suitable sensors to the workstation helps to recognize the right parts faster. Developments can be integrated with the AR solution to be even more effective in assembly work.

\section{Conclusion}

The use of AR creates opportunities to reduce the time spent on work. It is possible to follow the instructions faster. Usually, the guides are long and monotonous. Using conventional solutions, it is difficult for the compiler to read and monitor the assembly processes in text format when performing new tasks. The created application encompasses all the $3 \mathrm{D}$ models of the module by selecting precisely the right material to be displayed on the screen with 3D images, quantities and instructions. The project aimed to create an intelligent workplace for electro-technical sector factory, which has a kind of transformer cooling assembly. Based on analysed information of digital technology and considering the following factors: user-friendliness, energy efficiency, system logic and quality, the smart workstation should be quickly learnable and safer workplace. The results were found to be enhancing traceability and have a faster assembly process, increasing the quality, reducing cost and time, for example, reduction in the work. The application was completed and tested in a more straightforward form in the TalTech IVAR laboratory. The appearance of the application, user interface, the components animation, and optimisation of viewpoints were sufficient, and the feedback from the test users was positive. The test confirmed that the digitalisation of the manual work is an essential part as the user has interactive guides and 3D graphical models, which are giving a better idea of the assembly work. Further improvements could be the addition of more specific component properties into the application. Especially, add colour and a structure to further impart material 
authenticity. This symposium paper for DAAAM should encourage other industries to make prototypes with VR/AR applications which are suitable and improve work conditions. In the future, this AR prototype application goes to an assembly line where the industry can study and find additional needs.

\section{Acknowledgements}

Special thanks and gratitude to the students of Integrated Engineering program, Department of Mechanical and Industrial Engineering, Tallinn University of Technology.

\section{References}

[1] https://www.gartner.com/smarterwithgartner/5-trends-emerge-in-gartner-hype-cycle-for-emerging-technologies 2018/, (2018). Attard, M. "What Technologies Make the 2018 Gartner Hype Cycle?" Accessed: 2019-05-20.

[2] https://www.grandviewresearch.com/press-release/global-virtualprototype-vp-market, (2018). Grand View Research, "Virtual Reality Market Size Worth \$48.5 Billion By 2025.” Accessed: 2019-05-20.

[3] https://www.statista.com/statistics/591181/global-augmented-virtual-reality-market-size/, (2019). Statista, "Forecast augmented (AR) and virtual reality (VR) market size worldwide from 2016 to 2022 (in billion U.S. dollars)" Accessed: 2019-05-25.

[4] Mahmood, K.; Lanz, M.; Toivonen, V.\& Otto, T. (2018). A performance evaluation concept for production systems in an SME network. Procedia CIRP, 72, 603-608. DOI: https://doi.org/10.1016/j.procir.2018.03.182

[5] Zhong, R.Y.; Xu, X.; Klotz, E. \& Newman, S.T. (2017). Intelligent Manufacturing in the Context of Industry 4.0: A Review. Engineering, 3, 616-630. DOI: https://doi.org/10.1016/J.ENG.2017.05.015

[6] Karaulova, T.; Shevtshenko, E.; Polyanchikov, I.; Sahno, J. (2009). Reorganisation of Production System on SME Enterprises. Proceedings of the 20th DAAAM International Symposium: Intelligent Manufacturing \& Automation: Theory, Practice \& Education, 869 - 870. Published by DAAAM International.

[7] Cohen, Y.; Faccio, M.; Galizia, F.G.; Mora, C. \& Pilati, F. (2017). Assembly system configuration through Industry 4.0 principles: the expected change in the actual paradigms. IFAC PapersOnLine, 50, 14958-14963. DOI: https://doi.org/10.1016/j.ifacol.2017.08.2550

[8] Tamburini, D. (2018). "Augmented Reality becomes mainstream in Manufacturing, changes the face of the industry." Available from: https://azure.microsoft.com/enus/blog/augmented-reality-becomes-mainstream-inmanufacturing-changes-the-face-of-theindustry/ Accessed: 2019-05-25

[9] Novak-Marcincin, J.; Barna, J.; Janak, M. \& Novakova-Marcincinova, L. (2013). Procedia Computer Science, 25, 23-31. DOI: https://doi.org/10.1016/j.procs.2013.11.004

[10] Neb, A. \& Strieg, F. (2018). Generation of AR-enhanced Assembly Instructions based on Assembly Features, Procedia CIRP, 72, 1118-1123. DOI: https://doi.org/10.1016/j.procir.2018.03.210

[11] Mourtzis, D.; Samothrakis, V.; Zogopoulos, V. \& Vlachou, E. (2019). Warehouse Design and Operation using Augmented Reality technology: A Papermaking Industry Case Study. Procedia CIRP, 79, 574 - 579. DOI: https://doi.org/10.1016/j.procir.2019.02.097

[12] https://www.ptc.com/en/case-studies/volvo-group-digital-thread. (2019). "Volvo Group Delivers Digital Thread Through the Lens of Augmented Reality." PTC. Accessed: 2019-08-20.

[13] Mahmood, K.; Shevtshenko, E.; Karaulova, T.; Branten, E. \& Maleki, M. (2016). Troubleshooting Process Analysis and Development of Application for Decision Making Enhancement, Proceedings of the 26th DAAAM International Symposium, pp. 0663-0671, B. Katalinic (Ed.), Published by DAAAM International.

[14] Cognex, (2016). "Introduction to machine vision, a guide to automating process \& quality improvements.” Available from: https://www.assemblymag.com/ext/resources/White_Papers/Sep16/Introduction-to-Machine-Vision.pdf. Accessed: 2019-06-25

[15] Fraga-Lamas, P.; Fernandez-Carames, T. M.; Blanco-Novoa, O. \& Vilar-Montesinos, M. A. (2018). A Review on Industrial Augmented Reality Systems for the Industry 4.0 Shipyard. IEEE Access, vol. 6, pp. 13358-13375. DOI: 10.1109/ACCESS.2018.2808326

[16] Danielsson, O.; Syberfeldt, A.; Holm, M. \& Wang, L. (2018). Operators perspective on augmented reality as a support tool in engine assembly. Procedia CIRP, 72, 45 - 50. DOI: https://doi.org/10.1016/j.procir.2018.03.153

[17] https://program-ace.com/blog/microsoft-hololens-vs-hololens-2-comparison/. (2019). Program-Ace, "Microsoft HoloLens vs HoloLens 2 Comparison," Accessed: 2019-07-20.

[18] Kollatsch, C.; Schumann, M.; Klimant, P.; Wittstock, V. \& Putz, M. (2014). Mobile Augmented Reality Based Monitoring of Assembly Lines. Procedia CIRP, 23, 246 - 251.

[19] Okita, A. (2015). Learning C\# Programming with Unity 3D, CRC Press, Taylor \& Francis Group, Florida US.

[20] https://unity.com/solutions/mobile-ar. (2019). Unity, “Unity for mobile AR.” Accessed: 2019-07-20.

[21] Cushnan, D. \& Habbak, H.E. (2013). Developing AR Games for iOS and Android: develop and deploy augmented reality apps using Vuforia SDK and Unity 3D, Pack publishing, Birmingham, UK.

[22] https://engine.vuforia.com/engine. (2019). Vuforia engine, "Market-leading Augmented Reality Technology." Accessed: 2019-07-25 